\title{
Charmonium spectrum on dynamical anisotropic lattices
}

\section{K. Jimmy Juge*}

Trinity College, Dublin, Ireland

E-mail: juge@maths.tcd.ie

\section{Alan Ó Cais}

Trinity College, Dublin, Ireland

E-mail: alancemaths.tcd.ie

Mehmet B. Oktay ${ }^{\dagger}$

Trinity College, Dublin, Ireland

E-mail: bktay@maths.tcd.ie

\section{Mike J. Peardon}

Trinity College, Dublin, Ireland

E-mail: mjp@maths.tcd.ie

\section{Sinéad M. Ryan}

Trinity College, Dublin, Ireland

E-mail: ryan@maths.tcd.ie

We present a first study of the charmonium spectrum on $N_{f}=2$ dynamical, anisotropic lattices. We take advantage of all-to-all quark propagators to build spatially extended interpolating operators to increase the overlap with states not easily accessible with point propagators such as radially excited states of $\eta_{c}, \psi$ and $\chi_{c}$, D-waves and hybrid states.

XXIIIrd International Symposium on Lattice Field Theory

25-30 July 2005

Trinity College, Dublin, Ireland

\footnotetext{
*Speaker.

${ }^{\dagger}$ Based on talks by KJJ and MBO.
} 


\section{Introduction}

Many new states have been observed in the charmonium sector in the past few years. The missing spin singlet states as well as new, yet to be explained states such as the $\mathrm{X}(3872)$ [1], Y(4260)[2], $\mathrm{Y}(3940)[3]$ and others have presented a new challenge for the theoretical particle physics community.

Simulating the charmonium spectrum in lattice QCD has long been problematic due to the fact that the mass is too heavy for a simple relativistic action on coarse lattices and too light to expand in inverse powers of the quark mass. There are various quenched studies with improved actions which attempt to reduce lattice artifacts $\left[\begin{array}{l}4 \\ \text {, }\end{array}\right.$ but very little has been done on dynamical configurations [6].

In this study, we use a new dynamical, anisotropic action which has the advantage of being fully relativistic and yet has small mass-dependent discretization errors. All-to-all propagators [7] are utilized to make full use of the configurations and to construct extended operators for better overlap with the higher charmonium states.

\section{Dynamical anisotropic action}

The $N_{f}=2$ anisotropic quark action [8] is as follows:

$$
S_{q}=\bar{\psi}\left(\gamma_{0} \nabla_{0}+\sum_{i} \mu_{r} \gamma_{i} \nabla_{i}\left(1-\frac{1}{\xi_{q} a_{s}^{2}} \Delta_{i}\right)-\frac{r a_{t}}{2} \Delta_{i 0}+s a_{s}^{3} \sum_{i} \Delta_{i}^{2}+m_{0}\right) \psi
$$

where the links are fattened using stout links [9] which maximize the plaquette. The target renormalized anisotropy, $\xi$, is 6 . The nonperturbative tuning of the action has been presented elsewhere at this conference [10]. The sea quark mass was set near the strange quark in this first study although there are indications that there are no major problems simulating at lighter sea quark masses. The gauge action is the "two-plaquette" action [11] which was designed to reduce the cutoff effects for the scalar glueball on coarse anisotropic lattices. We use the same anisotropic fermion action for both light sea quarks and heavy valence quarks. In a quenched study [8], it was found that the same bare anisotropy can be used for a large range of quark masses. For the heavy valence quark, we have used both stout links and original links in this study.

\section{Operators}

We use a variational basis of operators, many of which are extended in space, to maximize the overlap with the higher charmonium states. All-to-all propagators which do not introduce large variance are crucial for this construction. We use the "dilution" method of Ref. [7] without eigenvectors for the charm quark propagators. Several dilution schemes were tested on a small number of configurations. We present results which use "time+colour+space-even-odd" dilution. Preliminary investigations indicate that even higher dilutions may further reduce the errors; this is work in progress.

The S-wave charmonium states only require different smearings of the quark to project out radially excited states. However, we have included a hybrid $1^{--}$operator which contains a gluonic 


\begin{tabular}{cc}
\hline Particle & Operator \\
\hline \hline $1^{--}$ & $\vec{\gamma}, \gamma_{5} \vec{u}, \gamma_{5} \vec{B}$ \\
$0^{-+}$ & $\gamma_{5}, \gamma_{5}\left(s_{1}+s_{2}+s_{3}\right)$ \\
$0^{++}$ & $\vec{\gamma} \cdot \vec{p}$ \\
$1^{++}$ & $\vec{\gamma} \times \vec{p}$ \\
$2^{++}$ & $\gamma_{k} p_{i}+\gamma_{i} p_{k}$ \\
$1^{+-}$ & $\gamma_{5} \vec{p}$ \\
$1^{-+}$ & $\vec{\gamma} \times \vec{u}, \vec{\gamma} \times \vec{B}$ \\
$2^{-+}$ & $\gamma_{5}\left(s_{1}-s_{3}\right), \gamma_{5}\left(2 s_{3}-s_{1}-s_{3}\right)$ \\
$3^{--}$ & $\vec{\gamma} \cdot \vec{t}$ \\
\hline
\end{tabular}

Table 1: Some operators used to project out various higher lying states. The notation for the gluonic paths is that of Ref. [12].

excitation in the form of a chromomagnetic and "staple" field. The operators used in this study are summarized in Table 1. Many of the operators used here resemble the ones used in Ref. [12]. In addition to these basic operators, we have used two different smearings for the quark fields to build a larger variational basis. The variational optimization was performed at the largest timeslice possible (with the metric timeslice fixed at $t=1$ ) where the results have become independent of this choice. We note that the $\eta_{c}$ and $\chi_{c}$ states (especially the $0^{++}$) required a large $t$ for the results to become stable. This may be an indication that better operators can improve the situation for these channels significantly.

For the exotic channel, $1^{-+}$, we have used both the staple and chromomagnetic field operator since we know that the staple operator has a large overlap with the first excited gluonic state in the static $q \bar{q}$ system [13]. The chromomagnetic field operator has the advantage of being an easy operator to construct as it is an operator at a single point. We have found that there is very little difference between the two operators, contrary to the non-relativistic quark model which suggests that the quarks are in a relative P-wave. This may be due to the fact that the quarks have been smeared or that both operators have a high level of excited state contamination from multi-particle states.

\section{Analysis}

\subsection{Fitting scheme}

The use of time-diluted all-to-all propagators makes it difficult to identify the plateau region (and judge its quality) from effective mass plots. This is due to the random noise introduced at each timeslice which makes locally measured quantities such as the effective mass fluctuate more than they would with point propagators. These "local fluctuations" do not affect exponential fits as long as a reasonable range of timeslices are included since these fits capture the long range exponential decay of the correlation function. (This feature will disappear once the gauge noise limit is reached in the dilution method. We already see a noticeable decrease of these effects at the higher dilution results that are presented here.) We therefore show instead " $t_{\min }$ " plots where fitted values of the 


\begin{tabular}{rcccc}
\hline Splitting & stout & $m_{0}$ & $a_{t} \Delta$ & $a_{t}^{-1}(\mathrm{GeV})$ \\
\hline \hline$\overline{\mathrm{P}}-\overline{\mathrm{S}}$ & with & 0.09 & $0.0568(5)$ & $8.06(7)$ \\
$\overline{\mathrm{P}}-\overline{\mathrm{S}}$ & w/o & 0.12 & $0.0668(7)$ & $6.85(7)$ \\
\hline
\end{tabular}

Table 2: Temporal lattice spacings determined from spin-averaged SP splittings in the charmonium sector for the two actions used in this study.

mass are shown against the minimum timeslice included in the single exponential fits. The largest timeslice to include in the fit was taken to be halfway across the lattice, if the fluctuations were not dominating the signal. We show a typical example of an effective mass $\left(1^{++}\right)$and its corresponding $t_{\min }$ plot on the left. Our criteria for selecting a fit is based on the $\chi^{2}$, fit range and the fit quality Q. We demand (although there could have been many other choices for the criteria for a "good fit") that $\chi^{2} / N_{d f}$ be less than 2, the quality of the fit be larger than 0.2 and that there is a range of $t_{\min }$ where these fit values are stable.
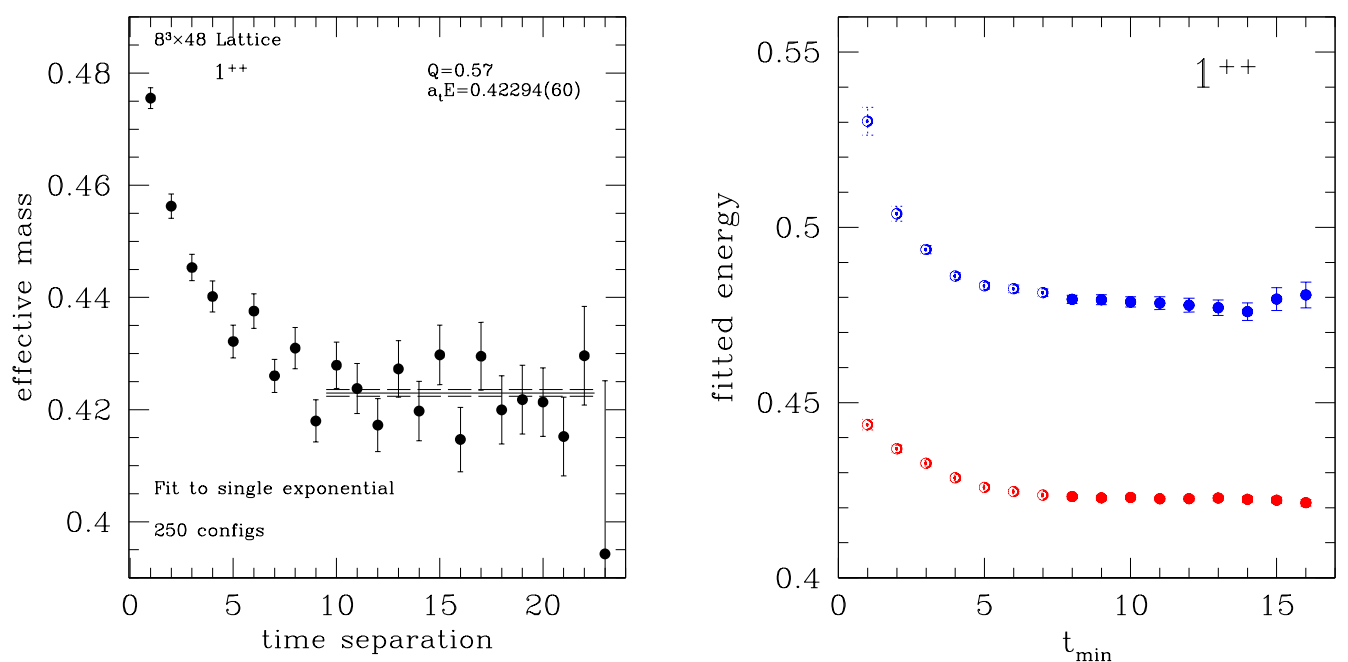

Figure 1: The effective mass of the $1^{++}$ground state is shown in the left figure. The " $t_{\min }$ plot" for this state is shown on the right. The open symbols in this plot indicate that the fit did not pass the requirements for a good fit (see text).

\subsection{Setting the scale}

We estimate the temporal lattice spacing from the S-P splitting in the charmonium sector. The results are tabulated in Table 2. We note that the bare quark masses were not quite tuned to the charm quark mass and the puzzling dependence on the mass and/or the effects of the stout-link is still under investigation.

\subsection{Dispersion relation}

In order to check that the $a M$ discretization errors are under control, we compute the dispersion relation for $\eta_{c}($ or $J / \psi)$ at various values of the momenta. The result is shown in Fig. 4.3. Since the 
renormalized anisotropy enters into this calculation, we cannot at this point determine unambiguously whether the kinetic mass is equal to the rest mass or not. This would require a determination of the dispersion relation of another state, e.g. $D_{s}$. We find a renormalized anisotropy determined at the charm quark mass which is significantly higher than what is found at the strange quark mass [14]. This discrepancy may be due to $a M$ errors, but it may also have its origins in the different methods used to determine the anisotropy (point propagators in [14] vs. all-to-all propagators here), combined with the short temporal extent of our lattice. This is currently under investigation, using lattices with a longer time extent.

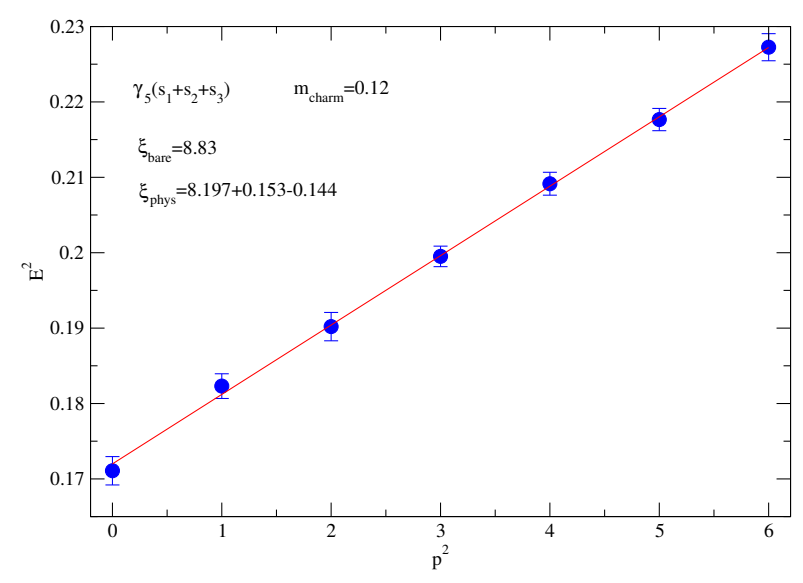

Figure 2: The dispersion relation computed for $\eta_{c}$ at a bare quark mass of 0.12 .

\section{Results and discussion}

The spectrum computed with (without) stout links is shown on the left (right) of Fig. 5. Since the quark mass has not been exactly tuned to the charm mass, the spectra are shown with the mass of the $J / \psi$ adjusted to its physical value. The main difference between the two spectra appears to be a change in the scale. Whether this is an artifact of the fitting criteria or the diagonalization procedure, or whether it is a real effect, is under investigation.

\subsection{Hyperfine splitting}

All previous lattice studies have given a too small value for the hyperfine splitting. It is clear that this problem is not yet resolved with this action. At this stage, the $\eta_{c}$ and $J / \psi$ are degenerate within the errors. There are several reasons why this splitting is not observed:

- Finite volume: The sides of this lattice are roughly $1.6 \mathrm{fm}$, which is quite small for charmonium. At this volume, finite temperature effects may also play a role [10].

- Higher order improvements of the action: The action used in this study has leading discretization errors of $\mathscr{O}\left(a_{t}, \alpha_{s} a_{s}, a_{s}^{3}\right)$. Higher order correction terms, in particular the $\sigma \cdot B$ term, may have a significant impact on the hyperfine splitting. 

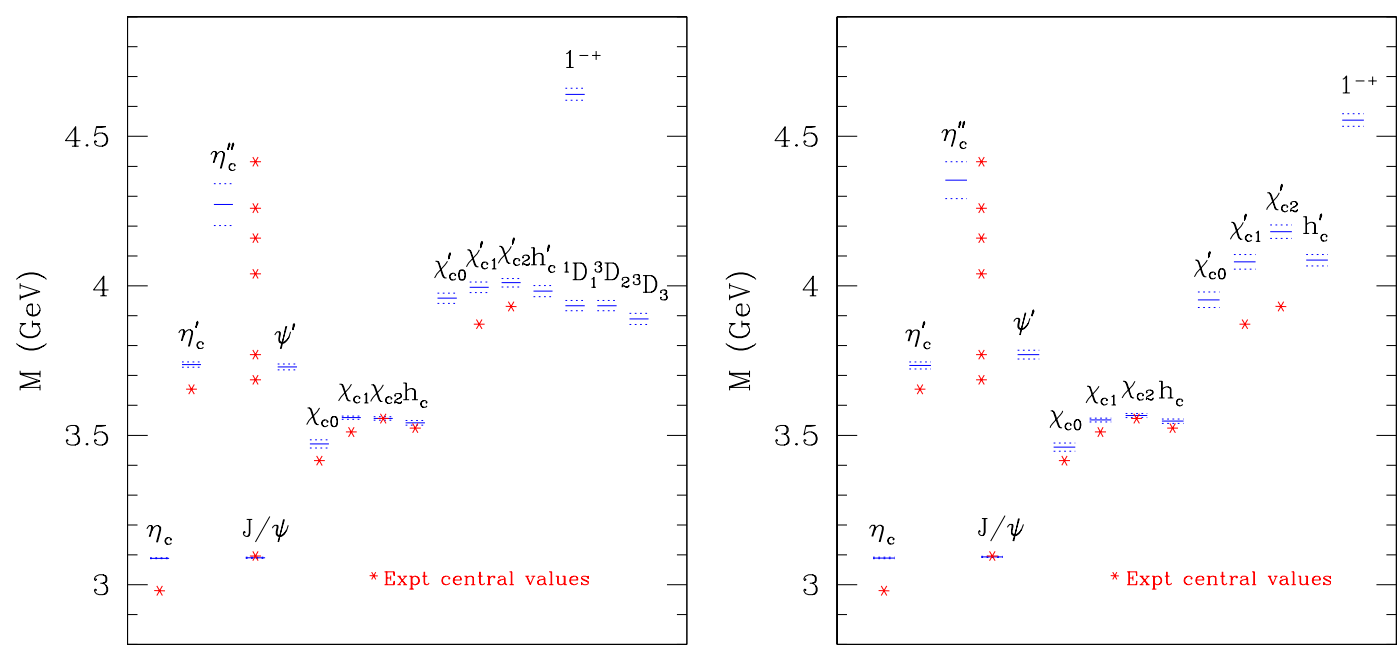

Figure 3: The charmonium spectrum from the simulation using stout links (left) and without stout links (right). The quantum numbers of some of the experimental findings for the higher lying P-waves have not been established yet; the assignment shown here is the one presented in Ref. [15].

- Light sea quark effects: The fact that the hyperfine splitting comes out too small in the quenched approximation indicates that light sea quark effects are important, and the sea quark mass that we are using may still be too large.

\subsection{States above threshold}

We have computed the masses of states which may lie above threshold, even at the relatively heavy sea quark mass used here. If this is so, then we must include multi-particle states in the spectrum and perform a finite volume analysis before we can claim that we have determined the energy of these states. Multi-particle operators are accessible with all-to-all quark propagators and investigations in this direction are under way.

Figure $\square$ shows the effective mass for the exotic hybrid $\left(1^{-+}\right)$, which is found to lie higher than in some quenched calculations (see [16] for example). For this case, however, it is probably even more important that a larger volume is used since the wavefunction of the exotic hybrid could be quite large [17] and would be squeezed in the small lattices used here.

\subsection{Hybrid content of $1^{--}$}

The correlation matrix element in the $1^{--}$channel between the state created using a standard $\bar{c} \vec{\gamma} c$ operator and a hybrid $\bar{c} \gamma_{5} \vec{B} c$ operator is plotted in Fig. 5. The coefficient appears to be zero within errors, suggesting that the hybrid content of the $\psi$ spectrum is small. The hybrid content of the $\Upsilon$ spectrum was also found to be small in a quenched calculation [18]. However, further reduction in noise is required before drawing any definitive conclusion.

\subsection{Disconnected diagrams}

Disconnected diagrams were not evaluated in this first study as these contributions are expected to be small (see [19] for example). However, the inclusion of these diagrams is not difficult 


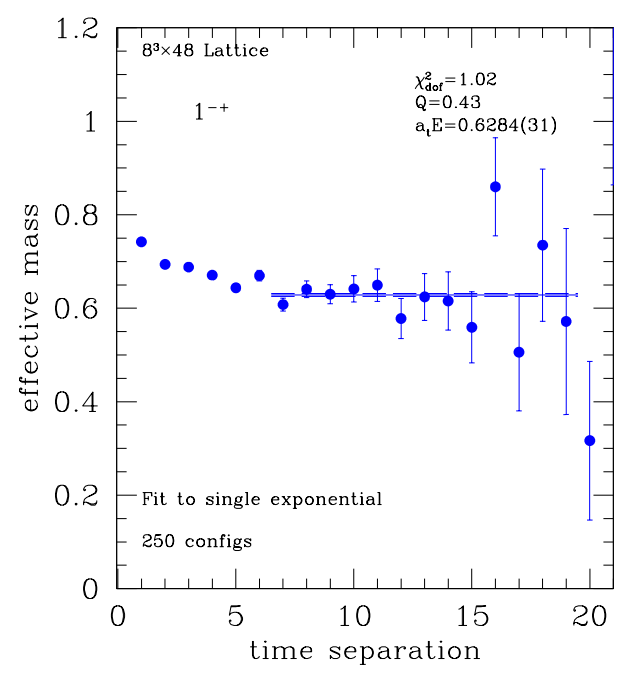

Figure 4: Effective mass of the $1^{-+}$.

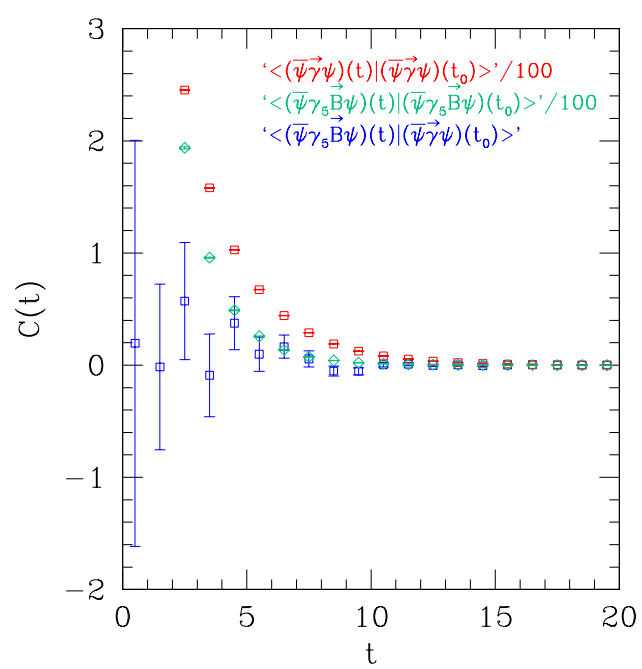

Figure 5: Correlation functions of the $1^{--}$state with different interpolating operators.

with the all-to-all propagators used here. A complete study with tuned parameters is planned to study the effect of these neglected diagrams on hyperfine splittings.

\section{Outlook}

A first study of the charmonium spectrum from an anisotropic, $N_{f}=2$ action was presented. All-to-all propagators and many operators were used to optimize the overlap with the standard states and obtain signals for higher lying radial excitations. A good signal for D-waves and exotic hybrid states was obtained with a relatively low level of dilution. However, short distance effects such as the hyperfine splittings require further investigation. A more detailed study on a larger volume and with more precisely tuned parameters is in progress. 


\section{Acknowledgements}

The authors would like to thank Jon-Ivar Skullerud for useful discussions. This work has been supported by the SFI grant 04/BRG/P0275 and the IITAC PRTLI initiative.

\section{References}

[1] Belle Collaboration, S. K. Choi et. al., Observation of a new narrow charmonium state in exclusive $B^{+-} \rightarrow K^{+-} \pi^{+} \pi^{-} J / \psi$ decays, Phys. Rev. Lett. 91 (2003) 262001 [hep-ex/0309032].

[2] BABAR Collaboration, B. Aubert et. al., Observation of a broad structure in the $\pi^{+} \pi^{-} J / \psi$ mass spectrum around $4.26 \mathrm{GeV} / \mathrm{c}^{2}$, hep-ex/0506081.

[3] Belle Collaboration, K. Abe et. al., Observation of a near-threshold omega J/ $\psi$ mass enhancement in exclusive $B \rightarrow K \omega J / \psi$ decays, Phys. Rev. Lett. 94 (2005) 182002 [hep-ex/ 0408126 .

[4] A. X. El-Khadra, The charmonium spectrum on the lattice: A status report, Nucl. Phys. Proc. Suppl. 30 (1993) 449-452 [hep-lat/9211046.

[5] CP-PACS Collaboration, M. Okamoto et. al., Charmonium spectrum from quenched anisotropic lattice QCD, Phys. Rev. $\mathbf{D 6 5}$ (2002) 094508 [hep-lat/0112020].

[6] M. di Pierro et. al., Properties of charmonium in lattice QCD with $2+1$ flavors of improved staggered sea quarks, Nucl. Phys. Proc. Suppl. 129 (2004) 340-342 [hep-lat/0310042].

[7] TrinLat Collaboration, J. Foley et. al., Practical all-to-all propagators for lattice QCD, Comp. Phys. Commun. (2005) [hep-lat/0505023]. in press.

[8] TrinLat Collaboration, J. Foley, A. Ó Cais, M. Peardon and S. M. Ryan, A non-perturbative study of the action parameters for anisotropic-lattice quarks, hep-lat/0405030.

[9] C. Morningstar and M. J. Peardon, Analytic smearing of SU(3) link variables in lattice QCD, Phys. Rev. D69 (2004) 054501 [hep-lat/0311018].

[10] R. Morrin et. al., Charmonium spectral functions in $N f=2$ QCD, PoS (LAT2005) 176 [hep-lat/0509115].

[11] C. Morningstar and M. J. Peardon, The glueball spectrum from novel improved actions, Nucl. Phys. Proc. Suppl. 83 (2000) 887-889 [hep-lat/9911003].

[12] UKQCD Collaboration, P. Lacock, C. Michael, P. Boyle and P. Rowland, Orbitally excited and hybrid mesons from the lattice, Phys. Rev. D54 (1996) 6997-7009 hep-lat/9605025].

[13] K. J. Juge, J. Kuti and C. Morningstar, Fine structure of the QCD string spectrum, Phys. Rev. Lett. 90 (2003) 161601 hep-lat/0207004.

[14] R. Morrin et. al., Tuning anisotropies for dynamical gauge configurations, PoS (LAT2005) 236.

[15] E. S. Swanson, Short range structure in the X(3872), Phys. Lett. B588 (2004) 189-195 [hep-ph/0311229].

[16] MILC Collaboration, C. W. Bernard et. al., Exotic mesons in quenched lattice QCD, Phys. Rev. D56 (1997) 7039-7051 hep-lat/9707008].

[17] K. J. Juge, J. Kuti and C. Morningstar, The heavy-quark hybrid meson spectrum in lattice QCD, AIP Conf. Proc. 688 (2004) 193-207 [hucl-th/0307116]. 
[18] MILC Collaboration, T. Burch and D. Toussaint, Hybrid configuration content of heavy S-wave mesons, Phys. Rev. D68 (2003) 094504 hep-lat/0305008.

[19] QCD-TARO Collaboration, P. de Forcrand et. al., Contribution of disconnected diagrams to the hyperfine splitting of charmonium, JHEP 08 (2004) 004 hep-lat/ 0404016 . 